\title{
Assessing Bioinspired Topographies for their Antifouling Potential Control Using Computational Fluid Dynamics (CFD)
}

\author{
Jacky Ling ${ }^{1}$ and Felicia Wong Yen Myan ${ }^{1, *}$ \\ ${ }^{1}$ Department of Mechanical Engineering, School of Engineering, Taylor's University, 1, Jalan \\ Taylors, 47500 Subang Jaya, Selangor, Malaysia.
}

\begin{abstract}
Biofouling is the accumulation of unwanted material on surfaces submerged or semi submerged over an extended period. This study investigates the antifouling performance of a new bioinspired topography design. A shark riblets inspired topography was designed with Solidworks and CFD simulations were antifouling performance. The study focuses on the fluid flow velocity, the wall shear stress and the appearance of vortices are to be noted to determine the possible locations biofouling would most probably occur. The inlet mass flow rate is 0.01 $\mathrm{kgs}^{-1}$ and a no-slip boundary condition was applied to the walls of the fluid domain. Simulations indicate that Velocity around the topography averaged at $7.213 \times 10^{-3} \mathrm{~ms}^{-1}$. However, vortices were observed between the gaps. High wall shear stress is observed at the peak of each topography. In contrast, wall shear stress is significantly low at the bed of the topography. This suggests the potential location for the accumulation of biofouling. Results show that bioinspired antifouling topography can be improved by reducing the frequency of gaps between features. Linear surfaces on the topography should also be minimized. This increases the avenues of flow for the fluid, thus potentially increasing shear stresses with surrounding fluid leading to better antifouling performance.
\end{abstract}

\section{Introduction}

The accumulation of unwelcomed biological organisms on a surface due to prolonged fluid flow is called biofouling. There are many industries that still face challenges because of this natural phenomenon [1]. Methods to prevent biofouling thus far have shown less than satisfactory effects. The nature and effects of biofouling come in different forms: biofouling in medical devices presents in layers of biofilms only; biofouling in marine and industrial applications contains layers of biofilm together with macrofouling and inorganic fouling. Macrofouling is similar as biofilm, however it is caused by the accumulation of macro sized organisms. Inorganic fouling on the other hand is caused by the accumulation of non-

* Corresponding author: feliciayenmyan.wong@taylors.edu.my 
biological substances, such as deposits from corrosion particles, crystallization, suspended particles, fuel, oil or ice [2].

Biofouling leads to many negative repercussions in the maritime industry and also could cause fatalities in the medical field. Biofouling greatly affects medical field from many common operations such as implants (prosthetic \& dental), blood transfusion, body organ sensors and any other medical operation involving interaction between a patient's body with the equipment. Medical equipment contaminated with biofouling material can be accidentally transferred to a patient leading to further illness or in more serious cases, death. In Malaysia, approximately 5000 deaths can be attributed to infections on medical equipment that were contaminated with biofouling material [3, 4].

Early efforts relied on toxic chemicals to deter biofouling. A well-known example is Tributylin (TBT) which has since been banned because of its harmful side effect towards marine life [5] Current research focuses on non-toxic approaches to deter biofouling and one such approach involves applies surface topographies. Field tests on various types of surface topographies should be supplemented by numerical studies to provide better evidences that surface topographies could be a sustainable antifouling approach.

Existing research on surface topographies focus on applying biomimicry to develop potential antifouling solutions. The inspiration for this research comes from observations of Self-cleaning abilities on some organisms allowing them to be free of the micro-organisms accumulation, lotus leaf and shark skin are both the common example of self-cleaning ability. Although the incorporation of biomimicry in antifouling purpose has only limited studies, but it has shown tremendous result in other relevant engineering applications. The most direct inspiration of nature towards antifouling would be from marine living organisms, although other source of inspiration should be viable too. Million years of evolution allowed some organisms to grow the ability to defend against harmful colonization on the hosts [6].

In numerical studies, computational fluid dynamics (CFD) is the sole approach to simulate the behavior of topographies regarding their abilities in antifouling. Through CFD, the fluid flow pattern and magnitude, together with other parameters such as vorticity and wall shear strain at specific location. These parameters are essential to determine the optimum design for better antifouling properties. This paper discusses studies on 3 different surface topographies inspired from the nature. The said parameters in each bio-inspired topography will be determined. Wall shear stress would be the main concern in deciding the antifouling performance because higher stress deny the movement of microbials, directly pushed them along the direction and prevent their localized settlements [7]. Input parameter such as fluid flow rate, viscosity, local environment condition plays the key role in the accuracy of results too and each of them will be discussed further.

\section{Research Methodology}

This research is conducted using CAD and CFD software due time and cost constraint, and thus field test will not be carried out.

\subsection{Selection of Bioinspired Topographies}

For this study, the inspiration for the bioinspired topography design came from combining features on several natural surfaces. This includes the textures found on shark's skin, lotus leaves and the pilot whale skin. All these surfaces possess natural self-cleaning abilities that prevents biofouling material from adhering to their surface so easily. 


\subsection{CAD 3D Model and CFD Setup}

After determining how the surface topographies should be like, a CAD 3D model for each topography is drawn on SolidWorks. The detailed dimension for each of the geometry in a model is justified to suit the size of simulation that is going to be carried out. CFD meshing is another crucial part in determining accurate result, a good mesh usually is complicated and time consuming, however it is much needed for a research like this, thus a mesh convergence analysis would be required after each simulation. This is to determine the best meshing quality and the accuracy of results generated.

\subsubsection{CAD Modelling}

Three dimensional models of the topographies were generated with SolidWorks. The geometry dimension of topography is as shown in Fig. 1.

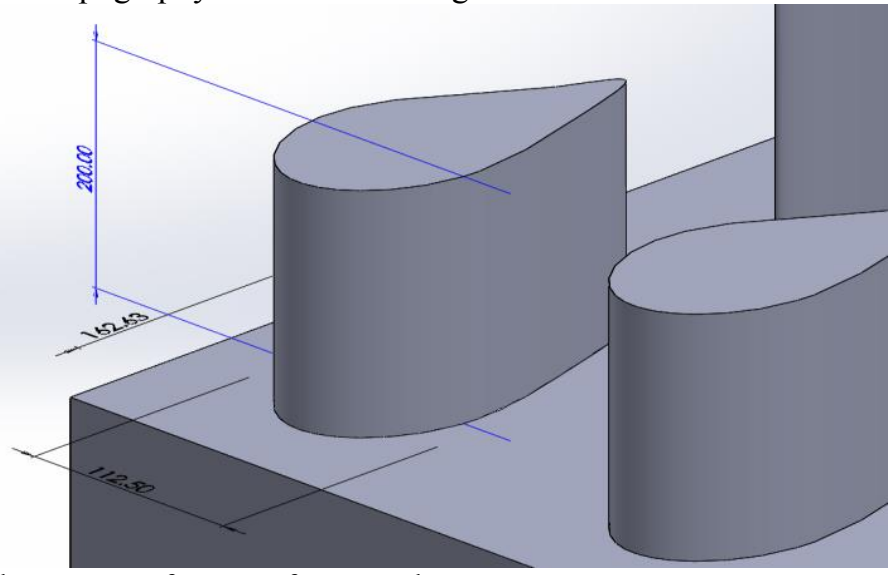

Fig. 1. Dimension of the geometry features of topography.

The geometry feature has width of $112.5 \mu \mathrm{m}, 162.63 \mu \mathrm{m}$ in length and $200 \mu \mathrm{m}$ in height. The gaps between each feature is $150 \mu \mathrm{m}$. The topography has 10 rows and column each on a $2 \mathrm{~mm} \times 3 \mathrm{~mm}$ plate that will be used later to create the fluid domain model for use in CFD. The fluid domain was represented by a solid block of $8 \mathrm{mmx} 8 \mathrm{mmx} 80 \mathrm{~mm}$ as shown in Fig. 2.

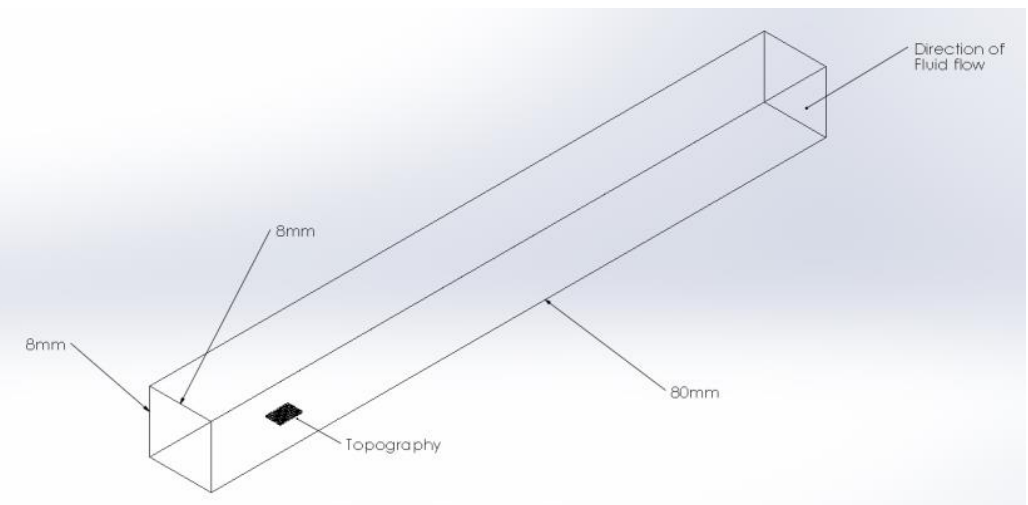

Fig. 2. Dimension of block representing fluid domain. 
The entire solid part will be detected as fluid domain automatically in CFD. The topography solid created earlier on will used to create cavity on the fluid domain block. The topography is placed at the bottom middle plane and $65 \mathrm{~mm}$ away from the inlet plane.

\subsubsection{Meshing Method}

ANSYS Workbench is used to create the meshing for the model. Firstly, the entire model was meshed with tetrahedral shape elements. This shape provides finer mesh around curvature and edges. Edge and face sizing was used at the edges and bottom plane respectively.

Wall shear stress is important in this simulation, thus inflation around the walls are created with 2 layers inflation of elements size $0.1 \mathrm{~mm}$. The resulting mesh are as shown in Fig. 5. Fig. 6 shows the close ups look at the meshing around the topography. To ease the setup of boundary conditions in CFD, the inlet, outlet and bottom wall topography was named respectively. This reduce the confusion when setting up boundary conditions, and makes previewing results easier.

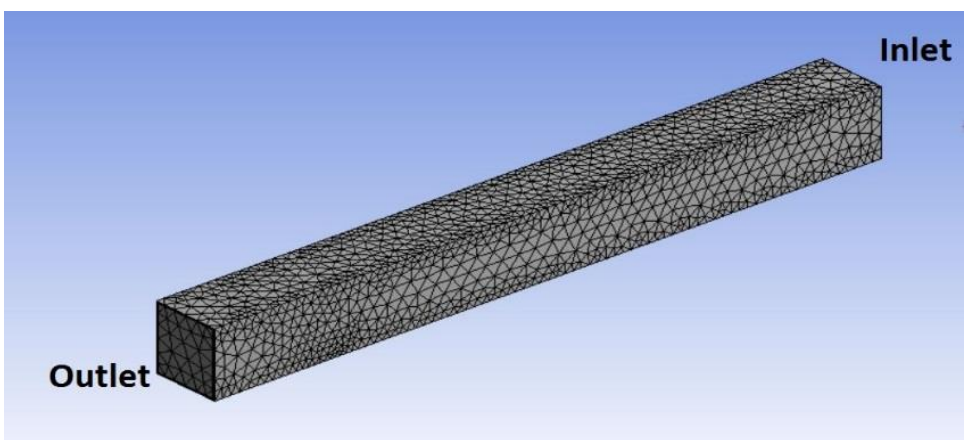

Fig. 5. Resulting mesh and indication of inlet and outlet.

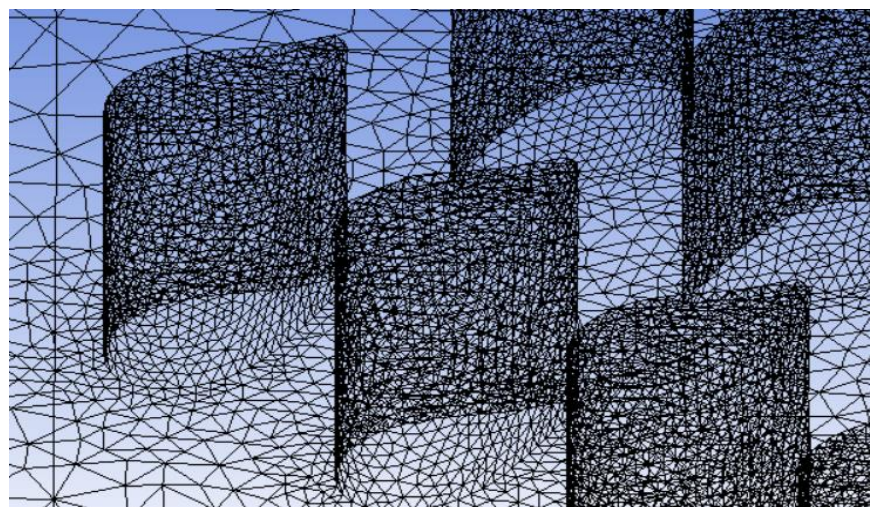

Fig. 6. Close-up look at the mesh around topography.

\subsubsection{Numerical Setup}

The use of Navier-Stokes equations (Equation 1 to 3 ) with the governing continuity equation (Equation 4) the mass and momentum to be conserved.

$$
\frac{\partial \rho \mathbf{u}}{\partial t}+\nabla \cdot \rho \mathbf{u} V=-\frac{\partial \rho}{\partial x}+\nabla \cdot(\mu \nabla \mathbf{u})+S_{m, x}
$$




$$
\begin{gathered}
\frac{\partial \rho \mathbf{v}}{\partial t}+\nabla \cdot \rho \mathbf{v} V=-\frac{\partial \rho}{\partial y}+\nabla \cdot(\mu \nabla \mathbf{v})+S_{m, y} \\
\frac{\partial \rho \mathbf{w}}{\partial t}+\nabla \cdot \rho \mathbf{w} V=-\frac{\partial \rho}{\partial z}+\nabla \cdot(\mu \nabla \mathbf{w})+S_{m, z} \\
\frac{\partial \rho \mathbf{u}}{\partial t}+\nabla \cdot(\rho V)=0
\end{gathered}
$$

The flow simulation required low Reynolds number formulation, transferred from a normal wall function, thus k-omega turbulence model was selected to ensure the accuracy of results. The density and dynamic viscosity of the fluid follows the default, which is 1000 $\mathrm{kgms}^{-3}$ and $0.001 \mathrm{kgm}^{-1} \mathrm{~s}^{-1}$ respectively. Fig. 7 shows the boundary condition applied on the model. No-slip boundary conditions were applied on the wall of fluid domain except inlet and outlet. Inlet mass flow rate was set at $0.001 \mathrm{kgs}^{-1}$ while the outlet was set as outflow.

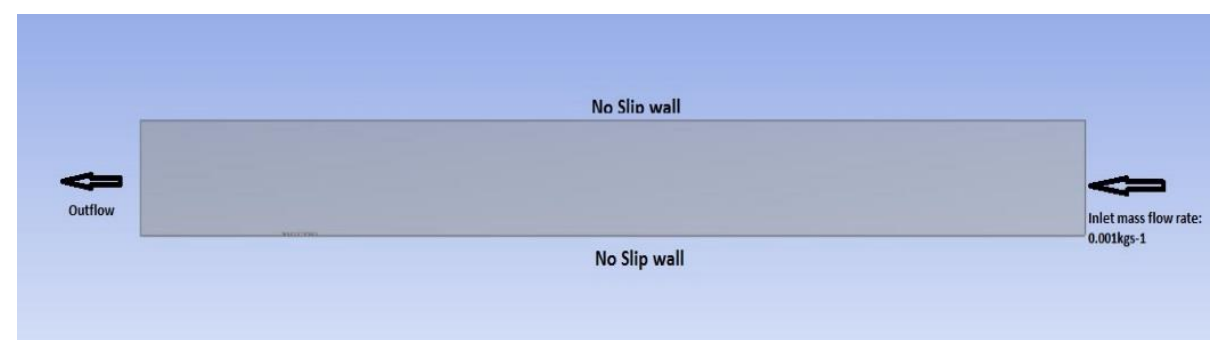

Fig. 7. Boundary conditions on the model.

\section{Results and Discussion}

\subsection{Mesh Convergence Analysis}

Mesh convergence analysis was carried out to determine the optimum number of mesh elements and assess the mesh quality. A few mesh refinements with different number of elements will be simulated and the difference in maximum wall shear stress will be compared to each other as shown in Table 1. The optimum number of elements is met when the difference in wall shear stress is less than $5 \%$.

Table 1. Mesh convergence at the model, mass flow rate at inlet $0.01 \mathrm{kgs}-1$.

\begin{tabular}{|c|c|c|c|}
\hline & & & $\begin{array}{c}\text { Optimum number of } \\
\text { elements }\end{array}$ \\
\hline $\begin{array}{c}\text { Mesh } \\
\text { refinements }\end{array}$ & $\begin{array}{c}1.4 \text { million to } \\
5.3 \text { million }\end{array}$ & $\begin{array}{c}5.3 \text { million to } \\
6.1 \text { million }\end{array}$ & \multirow{2}{*}{5.3 million } \\
\hline $\begin{array}{c}\text { Difference in } \\
\text { wall shear stress }\end{array}$ & $7.82 \%$ & $2.34 \%$ & \\
\hline
\end{tabular}

For this model, when the number of mesh element increased from 1.4 million to 5.3 million, the difference in wall shear stress exceeds $5 \%$, indicating the result are not stable and accurate enough. When the mesh refinement increased from 5.3 million to 6.1 million, the difference is only $2.34 \%$, this suggest that the results are nearly converged and further increase in number of elements would not give any big differences in the accuracy of results. 


\subsection{Velocity Streamlines and Vector Plot}

Velocity streamline and vector plot shows the fluid flow pattern around the topographies. Figure 8 presents the velocity streamline of fluid moving in between the topographies in the forward direction. The contour colours of the streamlines show that the velocity is increasing as the distance from the topographies increase in the $Y$ direction. ( $Y=4 \mathrm{~mm}$ ). The results indicate that fluid flow is slow around the topographies. The velocity above the topography $(\mathrm{Y}=0.2 \mathrm{~mm})$ is an average of only $7.213 \times 10^{-3} \mathrm{~ms}^{-1}$.

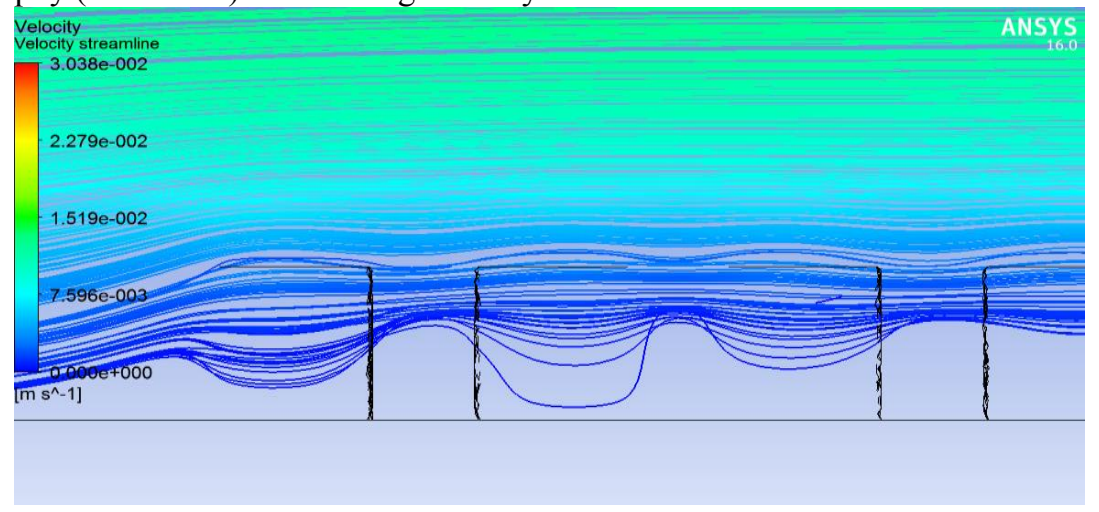

Fig. 8. Velocity streamline over topography.

Figure 9 presents the velocity vector profile between the gaps of Vortices were found in the flow direction, in between of subsequent topographies gap. The vortices formed and move in anti-clockwise direction (Fluid flow from right to left, in $\mathrm{Z}$ direction), preventing fluid from escaping the gap. This could also potentially trap the microorganisms in the gaps, allowing biofouling to occur.

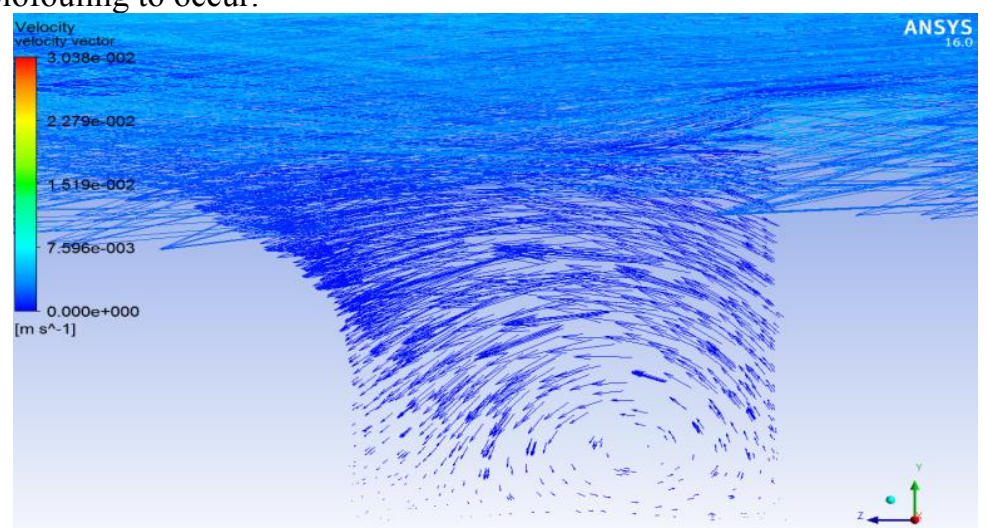

Fig. 91. Velocity vector plot showing vortices between the gaps of two adjacent feature in forward flow direction (right to left).

\subsection{Wall Shear Stress}

Several studies on biofouling have suggested that microorganisms have the ability to choose a suitable location for their settlements by adhesion. This indicates that surfaces that undergo higher wall shear would be more difficult for microorganisms to adhere to [8]. This indicates that high wall shear stress directly prevent biofouling. Fig. 10 presents the wall shear stress contour on one of the topography, viewing from side wall. 


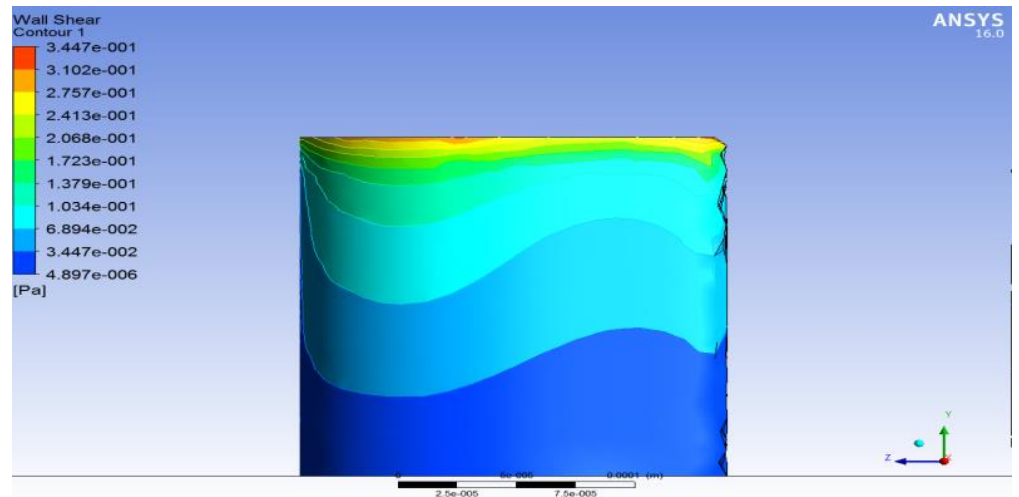

Fig. 10. Wall shear stress contour on the topography feature.

Fig. 11 presents the wall shear stress contour plot around the topographies at height of $100 \mathrm{um}$. Generally, the topographies have higher wall shear stress concentrated at the top, but significant low wall shear at the bed of topography. Highest wall shear stress appears at the tip of each topography, averaging at $0.231 \mathrm{~Pa}$. While the bed of topographies recorded an average $4.812 \times 10^{-6} \mathrm{~Pa}$. These values suggest that biofouling has the best chance to occur at the bed of topographies since the wall shear stress at the location are very weak.

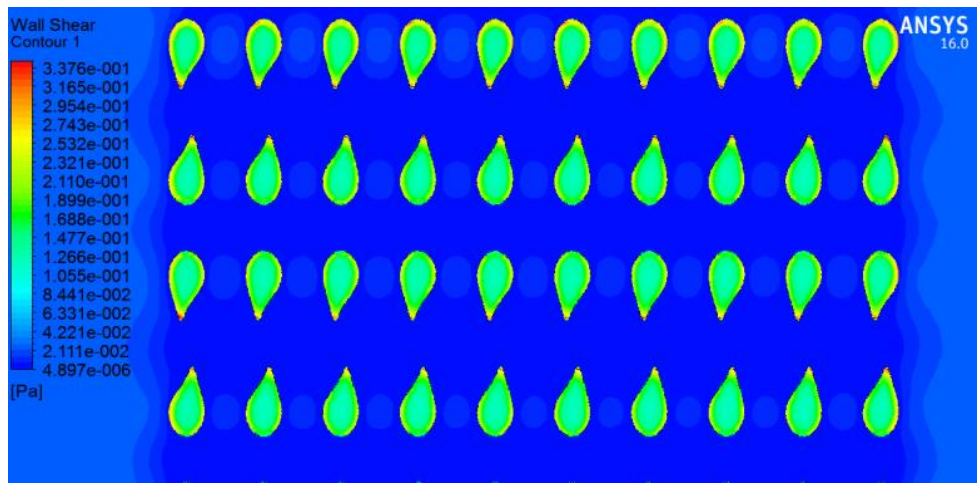

Fig. 11. Wall shear stress contour around the topography (100um above the bottom plane).

\section{Conclusion}

The numerical studies carried out determined the performance of bioinspired topographies against biofouling. The shark riblets inspired topography in this study exhibits average performance in antifouling based on the results collected in the form of velocity pattern and wall shear stress. The key problems to be noted from this study are as:

- The vortices formed in the gap of topographies

- Low wall shear stress at the bed of topographies

Further modifications on the topographies design aiming to eliminate the problem stated above should generate better results in term of antifouling performance.

\section{References}

1. Bixler, G. D. \& Bhushan, B. Biofouling: lessons from nature. Philos. Trans. R. Soc. 
370, 2381-2417 (2012).

2. A. I. Railkin, Ganf, T. A., O. G. Manylov, Marine biofouling colonization processes and defenses. Biofouling 20, 129-131 (2004).

3. J. Chan, S. Wong, Biofouling types, impact and anti-fouling. Biofouling types, impact and anti-fouling 1-70 (2010).

4. M. E. Shirtliff, J. G. Leid, in (eds. Shirtliff, M. \& Leid, J. G.) 3, 272 (Springer Berlin Heidelberg, 2009).

5. F. W. Y. Myan, J. Walker, O. Paramor, The interaction of marine fouling organisms with topography of varied scale and geometry: a review. Biointerphases 8, 27 (2013).

6. M. L. Carman et al. Engineered antifouling microtopographies - correlating wettability with cell attachment. Biofouling 22, 11-21 (2006).

7. P. Halder et al. A novel approach to determine the efficacy of patterned surfaces for biofouling control in relation to its microfluidic environment. Biofouling 29, 697713 (2013).

8. Y. K. Lee, Y. J. Won, J. H.Yoo, K. H. Ahn, C. H. Lee, Flow analysis and fouling on the patterned membrane surface. J. Memb. Sci. 427, 320-325 (2013). 